\title{
3-526-3
}

\section{Dynamics of HMGB1 in vascular endothelial cells and tumor vessels- targeting therapy using conjugated anti-HMGB1 mAb for cancers}

\author{
Masahiro Nishibori ${ }^{1}$, Shangze Gao ${ }^{1}$, Naoko Tomonobu², Masakiyo Sakaguchi ${ }^{2}$ \\ ${ }^{I}$ Dept of Pharmacol, Okayama Univ Grad Schl Med, ${ }^{2}$ Dept of Cell Biol, Okayama Univ Grad Schl Med
}

High mobility group box-1 (HMGB1), originally identified as a nuclear chromatin DNA-binding protein, plays a very important role in triggering the inflammatory responses in many kids of diseases once released into extracellular space. HMGB1 directly stimulates RAGE and TLR-4/2, and enhances the signal transduction by forming complexes with CXCL12 and IL-1beta. In the previous studies, we demonstrated that the treatment with anti-HMGB1 monoclonal antibody $(\mathrm{mAb})$ produced the beneficial effects on a diverse range of CNS and PNS diseases including brain ischemia, hemorrhage, trauma, epilepsy and neuropathic pain using animal models. During the studies, we noticed that the treatment with anti-HMGB1 mAb protected blood-brain barrier (BBB) from disruption very efficiently. Then, we focused on the distribution of therapeutic $\mathrm{mAb}$ in vivo and found that it accumulated around BBB in some types of brain injuries. To further investigate the dynamics of HMGB1 in vascular endothelial cells and the effects of anti-HMGB1 mAb, we used in vitro endothelial cell culture. We found that anti-HMGB1 mAb inhibited the stimulation-induced translocation of HMGB1, associated with the reduction of cytokine production. Surprisingly, anti-HMGB1 mAb was incorporated into endothelial cells considerably and we hypothesized that this incorporation could be applicable to DDS of anti-cancer drugs to tumor vessels. We will present in vivo data on the effects of conjugated $\mathrm{mAb}$ on melanoma in mice. 